\title{
PROPRIEDADES DE CHAPAS FABRICADAS COM PARTÍCULAS DE MADEIRA DE PARICÁ (Schyzolobium amazonicum Huber ex. Ducke) E FIBRAS DE COCO (Cocos nucifera L.) ${ }^{1}$
}

\begin{abstract}
Andrea Colli ${ }^{2}$, Benedito Rocha Vital ${ }^{3}$, Angêlica de Cássia Oliveira Carneiro ${ }^{3}$, José de Castro Silva ${ }^{3}$ Ana Márcia Macedo Ladeira Carvalho³ e Ricardo Marius Della Lucia ${ }^{3}$

RESUMO -Este trabalho teve como objetivo determinar as propriedades de chapas fabricadas com partículas de madeira de paricá (Schyzolobium amazonicum Huber ex. Ducke), às quais foram adicionadas diferentes proporções de fibras de coco (Cocos nucifera L.). As chapas foram fabricadas com 6 ou 8\% de adesivo, à base de ureia-formaldeído e tendo como meta uma massa específica de $360 \mathrm{~kg} / \mathrm{m}^{3}$. Observou-se que a adição de fibras de coco não afetou a estabilidade dimensional, higroscopicidade e absorção de água, porém aumentou significativamente as demais propriedades. As chapas fabricadas com $8 \%$ de adesivo foram mais estáveis e mais resistentes do que aquelas feitas com $6 \%$ de adesivo.
\end{abstract}

Palavras-chave: Aglomerado, Propriedades, Paricá e Fibra de coco.

\section{PROPERTIES OF PARTICLEBOARDS MADE OF PARICÁ (Schyzolobium amazonicum Huber ex. Ducke) PARTICLES AND COCONUT (Cocus nucifera L.) FIBERS}

\begin{abstract}
This work had as its objective to determine the properties of particleboards fabricated with paricá particles (Schyzolobium amazonicum Huber ex. Ducke), with increasing proportions of coconut fibers (Coconuts nucifera L.). Boards were fabricated with 6 or $8 \%$ of urea-formaldehyde adhesive. Particleboard mean density was equal to $360 \mathrm{~kg} / \mathrm{m}^{3}$. The addition of coconut fibers didn't affected boards'dimensional stability, higrospicidity, water absorption capability. However it increased its mechanical properties. The addition of $8 \%$ of adhesive improved all board properties.
\end{abstract}

Keywords: Particleboards, Properties, Paricá and Coconut fibers.

\section{INTRODUÇÃO}

De acordo com Zenid et al. (2004), os produtos derivados de madeira variam desde elementos com pouco ou nenhum processamento, como a madeira roliça, até aquela com vários graus de beneficiamento, como a madeira serrada e beneficiada, madeira preservada, lâminas e painéis de madeira. Segundo esses autores, os painéis de madeira surgiram da necessidade de amenizar as variações dimensionais da madeira maciça, diminuir seu peso e custo, bem como conservar as propriedades isolantes, térmicas e acústicas. Adicionalmente, suprem uma necessidade reconhecida no uso da madeira serrada e ampliam a sua superfície útil, através da expansão de uma de suas dimensões (largura), otimizando a sua aplicação.

Segundo Brito et al. (2004), a produção de chapas de partículas vem apresentando uma das maiores taxas de crescimento, entre os produtos à base de madeira. Isso está ocorrendo graças à diversidade de produtos disponíveis e da flexibilidade de aplicação para os mais variados fins. Os processos e a matériaprima utilizada em várias formas e geometrias, bem como os aditivos, conferem variação bastante ampla às propriedades do produto acabado.

\footnotetext{
${ }^{1}$ Recebido em 27.06.2008 e aceito para publicação em 14.10.2009.

${ }^{2}$ Mestre em Ciência Florestal, IEF. E-mail: <andreiacolli@hotmail.com>.

${ }^{3}$ Departamento de Engenharia Florestal, Universidade Federal de Viçosa, UFV, Brasil .E-mail: < bvital@ufv.br>, <cassiacarneiro@ufv.br>, <jcastro@ufv.br>,<ana.marcia@ufv.br>e<rdlucia@ufv.br>.
} 
Em princípio, todo e qualquer material lignocelulósico pode ser utilizado como matéria-prima para a fabricação de chapas de partículas, sendo a madeira o material mais usado. Geralmente, segundo Maloney (1993), madeiras de baixa densidade são preferidas porque permitem a fabricação de painéis mais leves e com propriedades adequadas.

Entre as inúmeras espécies de grande importância econômica na Amazônia, vem-se destacando o paricá (Schyzolobium amazonicum Huber ex. Ducke). Segundo Souza et al. (2005), a madeira é macia, leve, com textura grossa, grã-direita e irregular, cerne creme-avermelhado e alburno creme-claro. O processamento é fácil, e a baixa massa específica a indica como de bom potencial para a confecção de chapas de madeira aglomerada.

Além da madeira, outros materiais podem ser utilizados na composição das chapas. Maloney (1993) mencionou bagaço de cana, papiro, palha de cereais, talos de algodão, linho, bambu, papel e casca de coco. A escolha de determinada matéria-prima para a fabricação de chapas de composição depende do seu valor comercial, da disponibilidade e de algumas características intrínsecas, como densidade, cor e também o pH e a capacidade-tampão. Segundo Maloney (1993) e Medeved e Resnik (2004), o pH e a capacidade-tampão do material interferem na cura do adesivo, os quais geralmente são formulados para serem aplicados em material ligeiramente ácido e com baixa capacidade-tampão.

No Brasil, as iniciativas de aproveitamento das cascas de coco verde são tímidas, mesmo sendo consideradas matérias-primas com alta viabilidade de aproveitamento. Segundo Passos (2005), as cascas de coco são costumeiramente destinadas aos aterros e vazadouros, sendo potencial emissoras de metano, como toda matéria orgânica. Embora seja tratada como resíduo, é material que produz fibra com características peculiares, podendo ser usada na formulação de compósitos de grande valor industrial. As fibras naturais possuem baixa massa específica e podem melhorar algumas propriedades dos materiais, tal como a sua perspirabilidade. Por isso, a sua utilização como material de reforço em compósitos vem sendo cada vez mais pesquisada como alternativa para as matérias-primas tradicionais (SOUZA, 1999).

O objetivo deste trabalho foi determinar as propriedades das chapas de partículas aglomeradas fabricadas com madeira de paricá (Schyzolobium amazonicum Huber ex. Ducke) e fibras de coco (Cocos nucifera L.), em diferentes proporções e teores de adesivo, à base de ureia-formaldeído.

\section{MATERIAL E MÉTODOS}

A madeira de paricá(Schizolobium amazonicum Huber Ducke) foi obtida de árvores colhidas em plantios comerciais com idades de 5, 7, 9 e 11 anos, do Centro de Pesquisa do Paricá (CPP), no Município de Dom Eliseu, no Estado do Pará. As toras foram desdobradas em tábuas com 25 mm de espessura e as partículas de madeira, preparadas a partir de maravalhas, produzidas numa desengrossadeira, a partir de tábuas retiradas da região próxima da casca. As maravalhas foram, posteriormente, fragmentadas num moinho-martelo. Os finos foram removidos empregando-se uma peneira com $10 \mathrm{~mm}^{2}$ de abertura, o que resultou um índice de esbeltez médio igual a 7,09.

As fibras de coco (Cocos nucifera L.) foram fornecidas pela empresa DEFLOR (Belo Horizonte) sob a forma de manta, que foi desfeita, sendo as fibras fragmentadas em um moinho de martelo, com a obtenção de um índice de esbeltez médio de 36,66.

As partículas foram inicialmente secas ao ar até cerca de $15 \%$ de unidade e, posteriormente, secas em estufa à temperatura de $45 \pm 2{ }^{\circ} \mathrm{C}$ até um teor médio de umidade igual a $3 \%$.

Para determinação do $\mathrm{pH}$, modificou-se a metodologia descrita por Vital (1973), que consistiu em secar em estufa a $(103 \pm 2)^{\circ} \mathrm{C}$ partículas finas da madeira de paricá e da fibra de coco até peso constante. A seguir foram pesadas amostras contendo $15 \mathrm{~g}$ do material, que ficaram imersos em $150 \mathrm{~mL}$ de água destilada por $30 \mathrm{~min}$, à temperatura de $22^{\circ} \mathrm{C}$. Em seguida, foram filtrados em vácuo e $50 \mathrm{~mL}$ do líquido resultante, usado para determinação do $\mathrm{pH}$, empregando-se um pHmetro digital. Foram realizadas medições no extrato puro e após a adição de 1,0 mL de uma solução de hidróxido de sódio $(\mathrm{NaOH})$, em concentração de 0,025 molar, até um máximo de cinco adições.

Empregou-se adesivo, à base de ureia-formaldeído (Cascamite), doado pela Alba Adesivos e cujas propriedades são apresentadas no Tabela 1. O teor de sólidos foi calculado de acordo com Carneiro (2006), evaporando-se a água das amostras contendo $3 \mathrm{~g}$ de adesivo, que foram levados à estufa até peso constante. Para determinação do $\mathrm{pH}$, utilizaram-se cerca de 15 g do adesivo; o tempo de gelatinação foi obtido com a adição de 3 g do adesivo, num tubo de ensaio; já acrescentado o catalisador, foi inserido num frasco 
Tabela 1 - Propriedades do adesivo à base de ureia-formaldeído. Table 1 - Urea-formaldehyde based adhesive properties.

\begin{tabular}{lc}
\hline \multicolumn{2}{c}{ Características do adesivo } \\
\hline Teor de sólidos (\%) & 65 \\
pH & 7,97 \\
Viscosidade (Cp) & 540 \\
Tempo de gelatinização (segundos) & 58 \\
\hline
\end{tabular}

contendo óleo de silicone aquecido a $170{ }^{\circ} \mathrm{C}$ e agitado continuamente com um bastão de vidro até que o adesivo se desprendesse das paredes do tubo de ensaio. A viscosidade foi determinada utilizando-se amostras de 100 g do adesivo e empregando um viscosímetro de Brookfield (cp), com haste (Spindler) número 3, velocidade de 12 rpm e fator de conversão 100.

As chapas foram fabricadas com dimensões nominais de 60 x 60 x $1 \mathrm{~cm}$, utilizando-se porcentagens variadas de partículas de paricá (100, 90, 80 e 70\%) e fibras de coco $(0,10,20$ e $30 \%)$ e com 6 ou $8 \%$ de adesivo na base seca, conforme apresentado no Tabela 2 .

Teve-se como meta produzir chapas de baixa densidade, com massa específica de $360 \mathrm{~kg} / \mathrm{m}^{3}$, a uma taxa de compactação igual a 1,3/1,0. Foram fabricadas 24 chapas com três repetições em cada tratamento, totalizando 24 unidades.

Para o preparo das chapas, as partículas foram colocadas num encolador para a mistura de partículas com o adesivo, empregando-se uma pistola pneumática. O colchão foi formado manualmente, fazendo-se uma pré-prensagem, com o objetivo de lhe dar compactação mínima e que permitisse a sua inserção na prensa. A prensagem propriamente dita foi realizada com uma prensa mecânica, de pratos planos, na temperatura de $170{ }^{\circ} \mathrm{C}$ e pressão de $32 \mathrm{kgf} / \mathrm{cm}^{2}$.

Tabela 2 - Percentagem de partículas de madeira de paricá, fibra de coco e teores de adesivo empregados na fabricação das chapas de aglomerado.

Table 2 - Percentage of paricá wood particles, coconut fiber and adhesive content used to produce particleboards.

\begin{tabular}{cccc}
\hline Tratamento & $\begin{array}{c}\text { Teor de } \\
\text { adesivo (\%) }\end{array}$ & $\begin{array}{c}\text { Partículas de } \\
\text { paricá (\%) }\end{array}$ & $\begin{array}{c}\text { Fibras de } \\
\text { coco (\%) }\end{array}$ \\
\hline T 1 & 6 & 100 & 00 \\
T 2 & 8 & 100 & 00 \\
\hline T 3 & 6 & 90 & 10 \\
T 4 & 8 & 90 & 10 \\
\hline T 5 & 6 & 80 & 20 \\
T 6 & 8 & 80 & 20 \\
\hline T 7 & 6 & 70 & 30 \\
T 8 & 8 & 70 & 30 \\
\hline
\end{tabular}

Uma vez fabricadas, as chapas foram climatizadas por um período de $72 \mathrm{~h}$ para, então, serem confeccionados os corpos-de-prova, que foram acondicionados em câmara climática até atingir a umidade de equilíbrio, nas condições de $65 \pm 5 \%$ de umidade relativa e temperatura de $25 \pm 3^{\circ} \mathrm{C}$, segundo a norma ABNT 2002 _ NBR 14810-1/2002.

A densidade, resistências à tração perpendicular, flexão estática e arranque de parafuso, dureza JANKA, teor de umidade, expansão linear, inchamento e absorção de água foram determinadas seguindo-se os procedimentos estabelecidos na norma ABNT 2002 _ NBR 14810.

O experimento foi instalado segundo delineamento inteiramente casualizado, com quatro proporções de fibras de coco e dois teores de adesivo, com três repetições, totalizando 24 chapas. Os efeitos dos tratamentos nas propriedades das chapas foram analisados com o auxílio de uma análise de variância (ANOVA). Quando estabelecidas diferenças significativas, os tratamentos foram comparados entre si, por meio do teste de Tukey a 5\% de significância. Utilizou-se o software SAEG.

\section{RESULTADOS E DISCUSSÃO}

Conforme pode ser observado nas Figuras 1 e 2, o extrato da madeira de paricá apresentou $\mathrm{pH}$ médio de 4,88 e as fibras de coco, 5,70. Tanto a madeira quanto a fibra de coco tiveram baixa capacidade-tampão, uma vez que a adição de apenas $1 \mathrm{~mL}$ do $\mathrm{NaOH}$ foi suficiente para elevar o pH para 8,20 e 8,60, respectivamente. Portanto, não foi necessário o emprego de quantidades adicionais de catalisador no adesivo.

Após o equilíbrio, a uma temperatura de $25^{\circ} \mathrm{C}$ e $65 \%$ de umidade relativa, as chapas apresentaram teor de umidade médio de 14\%. Essa média superior àquela encontrada por Imamura et al. (1989) (9,4\%) em aglomerado fabricado com partículas de albizzia (Albizia falcata Backer), espécie com densidade similar à do paricá, porém próxima aos valores observados por Silva et al. (2005) em aglomerado industrial.

A massa específica média das chapas foi de $360 \mathrm{~kg} / \mathrm{m}^{3}$, sendo, portanto, classificadas como chapas de baixa densidade, segundo a norma NBR 14810-1/ 2002 da ABNT. Não foram observadas diferenças significativas na massa específica em função da composição das partículas ou dos teores de adesivo.

R. Árvore, Viçosa-MG, v.34, n.2, p.333-338, 2010 


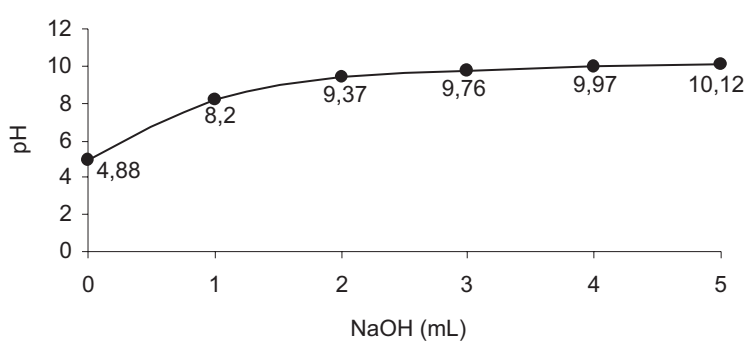

Figura 1 - Variação no pH da madeira de paricá (Schizolobium Amazonicum Huber ex. Ducke) em função da adição de uma solução de NAOH a 0,025 molar.

Figure 1 - Variation in the pHof the paricá wood (Schizolobium Amazonicum Huber ex. Ducke) with the addition of a NAOH 0,025 mole solution.

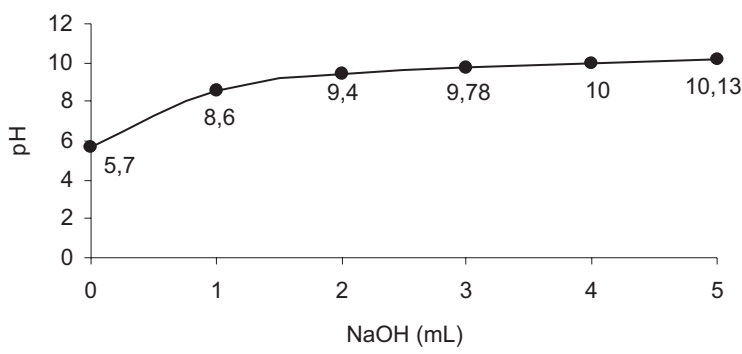

Figura 2 - Variação no pH o da fibra de coco (Cocos nucifera L.). Figure 2-Variation in the $\mathrm{pH}$ of the coconut fiber (Cocos nucifera L.).

A adsorção média de água após equilíbrio, a uma temperatura de $25^{\circ} \mathrm{C}$ e $65 \%$ de umidade relativa, é apresentada no Tabela3. Observa-se, nesse quadro, que a inclusão de $30 \%$ de fibra de coco ocasionou aumento significativo na umidade de equilíbrio higroscópico, independentemente do tipo de adesivo. Nas chapas fabricadas com $6 \%$ de adesivo, a menor higroscopicidade foi observada naquelas contendo 10 e $20 \%$ de fibras de coco. No entanto, as chapas com $8 \%$ de adesivo e 0,10 ou $20 \%$ de fibra de coco adsorveram menos água do que aquelas contendo 305 de fibra.

Tabela 3 - Umidade média de equilíbrio higroscópico em função do teor de adesivo e da composição das partículas.

Table 3 - Equilibrium moisture content as affected by adhesive content and particles composition.

\begin{tabular}{ccccr}
\hline Teor de & \multicolumn{4}{c}{ Composição - Percentagem de fibras de coco } \\
\cline { 2 - 5 } adesivo $\%$ & 0 & 10 & 20 & 30 \\
\hline 6 & $14,87 \mathrm{a} \mathrm{B}^{*}$ & $12,74 \mathrm{aC}$ & $14,01 \mathrm{aBC}$ & $17,51 \mathrm{aA}$ \\
8 & $13,47 \mathrm{bAB}$ & $12,94 \mathrm{aB}$ & $13,99 \mathrm{aAB}$ & $14,24 \mathrm{bA}$ \\
\hline
\end{tabular}

*Médias seguidas pela mesma letra maiúscula ao longo da mesma linha ou pela mesma letra minúscula ao longo da mesma coluna não diferem entre si, pelo teste de Tukey a 5\% de probabilidade.
Considerando a média geral, observa-se, no Tabela 4, que aumento no teor de fibra ocasionou redução significativa na higroscopicidade das chapas.

A expansão linear média das chapas após o equilíbrio, com umidades relativas iguais a 65 e $90 \%$ e temperatura igual a $25^{\circ} \mathrm{C}$, foi igual a $0,49 \%$. Esse valor é inferior àquele permitido em chapas de aglomerado, segundo a especificação da American National Standard for Particleboard A 208.1 da NPA (1993). A análise de variância mostrou que não houve efeito significativo da composição das partículas e da interação entre a composição e o teor de adesivo nessa propriedade. Observaram-se, no entanto, diferenças significativas em função do teor de adesivo (Tabela 4); as chapas fabricadas com $6 \%$ de adesivo apresentaram expansão linear média de $0,52 \%$,valor significativamente superior ao das chapas fabricadas com $8 \%$ de adesivo, cuja expansão linear média foi igual a 0,44\%.

Os valores médios de inchamento em espessura das chapas, após 2 e 24 h de imersão em água, foram iguais a $10,2 \%$ e $11,79 \%$, respectivamente. Observou-se que, num período curto de imersão ( 2 h), as chapas apresentaram inchamento próximo daquele obtido após a imersão de 24 h. Apesar da grande absorção de água, o inchamento em espessura foi inferior ao limite máximo permitido pela norma (DIN 68761 (1)-1961. Não se observou diferença significativa em função da composição das partículas, nem interação entre a composição e os teores de adesivo utilizados. Para ambos os tempos de imersão em água, no entanto, verificou-se que as chapas fabricadas com $8 \%$ de adesivo foram mais estáveis do que aquelas com 6\%.

A composição das partículas não afetou significativamente a absorção de água, após 2 ou 24 h de imersão. A absorção média de água foi igual a 194\% e 220\% após 2 e 24 h de imersão em água, respectivamente. Portanto, houve elevada absorção de água em curto período de imersão. No entanto, a absorção de água foi afetada pelo teor de adesivo. As chapas fabricadas com $8 \%$ de adesivo absorveram significativamente menos água do que aquelas fabricadas com 6\%. Aumento na estabilidade dimensional ocasionado pelo aumento no teor de adesivo tem sido observado por alguns autores (MALONEY, 1993; VITAL e HASELEIN, 1988).

Os valores médios das propriedades mecânicas, em função dos tratamentos, estão apresentados no Tabela 5. O módulo de ruptura em flexão foi afetado 
Tabela 4-Umidade de equilíbrio higroscópico, absorção de água, expansão linear e inchamento em espessura em função do tempo de imersão em água e do teor de adesivo.

Table 4 - Equilibrium moisture content, linear expansion and thickness swelling as affected by immersion time and adhesive content.

\begin{tabular}{cccccc}
\hline Teor de adesivo & \multicolumn{5}{c}{ Propriedades } \\
\cline { 2 - 5 } & UE & EL & I2H & I24H & A24H \\
\hline 6 & $14,78 \mathrm{a}^{*}$ & $0,52 \mathrm{a}$ & $11,26 \mathrm{a}$ & $13,11 \mathrm{a}$ & $228,50 \mathrm{a}$ \\
8 & $13,66 \mathrm{~b}$ & $0,48 \mathrm{a}$ & $9,12 \mathrm{~b}$ & $10,46 \mathrm{~b}$ & $211,83 \mathrm{a}$ \\
\hline
\end{tabular}

*Médias seguidas pela mesma letra minúscula ao longo da mesma coluna não diferem entre si, pelo teste de Tukey a 5\% de probabilidade.

Em que EU = umidade de equilíbrio higroscópico; A24H = absorção de água após 24 h de imersão; EL = expansão linear após 2 h de imersão em água; e I2H, I24H = inchamento em espessura após 2 e 24 h de imersão em água, respectivamente.

Tabela 5 - Médias da propriedades mecânicas das chapas em função da composição das partículas e do teor de adesivo. Table 5 - Boards mechanical properties means as affected by particles composition and adhesive content.

\begin{tabular}{cccccr}
\hline $\begin{array}{c}\text { ComposiçãoPercentagem } \\
\text { de fibra de coco }\end{array}$ & \multicolumn{5}{c}{ Propriedades } \\
\cline { 2 - 6 } & TRAÇ̃̃O(MPa) & MOR(MPa) & MOE(MPa) & DUREZA(MPa) & PARAFUSO(N) \\
\hline 0 & $0,22 \mathrm{a} *$ & $2,38 \mathrm{~b}$ & $160,5 \mathrm{a}$ & $9,29 \mathrm{a}$ & $230,75 \mathrm{a}$ \\
10 & $0,16 \mathrm{ab}$ & $3,43 \mathrm{~b}$ & $240,0 \mathrm{a}$ & $12,03 \mathrm{a}$ & $249,77 \mathrm{a}$ \\
20 & $0,13 \mathrm{~b}$ & $3,99 \mathrm{ab}$ & $244,6 \mathrm{a}$ & $14,55 \mathrm{a}$ & $275,60 \mathrm{a}$ \\
30 & $0,18 \mathrm{ab}$ & $4,46 \mathrm{a}$ & $255,6 \mathrm{a}$ & $13,76 \mathrm{a}$ & $294,98 \mathrm{a}$ \\
\hline Teor de adesivo\% & & & & $240,20 \mathrm{~b}$ \\
\hline 6 & $0,15 \mathrm{~b}$ & $3,00 \mathrm{~b}$ & $191,8 \mathrm{~b}$ & $11,53 \mathrm{a}$ & $295,80 \mathrm{a}$ \\
\hline
\end{tabular}

*Médias seguidas pela mesma letra minúscula ao longo da mesma coluna não diferem entre si, pelo teste de Tukey a 5\% de probabilidade.

pela composição das partículas e teor de adesivo. A adição de fibras de coco, de modo geral, ocasionou aumento no módulo de ruptura, sendo as chapas mais resistentes aquelas contendo 20 ou 30\% de fibras. As chapas fabricadas com $8 \%$ de adesivo apresentaram valor médio de módulo de ruptura (3,0 MPa) superior àqueles encontrados nas chapas com $6 \%$ de adesivo (4,1 MPa). O módulo de elasticidade não foi afetado pela composição das partículas. Contudo, o teor de adesivo afetou significativamente essa propriedade. As chapas fabricadas com $6 \%$ de adesivo apresentaram um MOE de 191 MPa e as com 8\% de adesivo, MOE de $258 \mathrm{MPa}$. Devido à elevada correlação entre o módulo de elasticidade e a resistência à ruptura observada por alguns autores, esperava-se que as diferenças no MOE decorrentes da composição das partículas fossem significativas, contudo isso não ocorreu.

A resistência à tração perpendicular foi afetada tanto pelo teor de adesivo quanto pela porcentagem de fibra de coco. As chapas produzidas com 6 e $8 \%$ de adesivo apresentaram valores de resistência média à tração de 0,21 Mpa e 0,15 Mpa, respectivamente. No que tange à composição das partículas, os tratamentos que continham fibra de coco, em geral, apresentaram menor resistência à tração do que os tratamentos que mostravam somente partículas de paricá. Isso indica que as fibras de coco prejudicaram a adesão entre os componentes das chapas.

A resistência das chapas ao arrancamento de parafuso foi afetada apenas pelo teor de adesivo, em que as chapas produzidas com $8 \%$ de adesivo apresentaram resistência de 295,8 N, enquanto as confeccionadas com 6\%, de 240,2 N. Observou-se, ainda, tendência de aumento numérico na resistência ao arrancamento de parafuso com o acréscimo de fibra de coco. Contudo, as diferenças não foram significativas.

A dureza Janka das chapas não foi afetada pelos tratamentos. Os valores médios de dureza encontrados foram de 12,04 Mpa, com uma tendência de aumento

R. Árvore, Viçosa-MG, v.34, n.2, p.333-338, 2010 
desses valores com o aumento da porcentagem de fibra de coco adicionada à mistura com partículas de madeira na composição.

\section{CONCLUSÕES}

A madeira de paricá é apropriada para confecção de chapas aglomeradas, obtendo-se melhores propriedades quando fabricadas com $8 \%$ de adesivo.

A inclusão de fibras de coco permite melhorar algumas propriedades mecânicas das chapas.

\section{AGRADECIMENTOS}

Ao Centro de Pesquisa do Paricá (CPP) e aos engenheiros Luciano Zaneti e Marco Antônio Sivieiro, pelo auxílio na obtenção da madeira de paricá; à DEFLOR, pelo fornecimento das fibras de coco; e à Alba Adesivos, pelo fornecimento do adesivo.

\section{REFERÊNCIAS}

AMERICAN NATIONAL STANDARD - ANS. Mat-formed wood particleboard: specification. ANSI/A 208.1. 1993. Gaithersburg: National Particleboard Association, 1993. 9p.

\section{ASSOCIAÇÃO BRASILEIRA DE NORMAS} TÉCNICAS - ABNT. NBR-14810-1: Chapas de madeira aglomerada Rio de Janeiro: 2002. 32p.

BRITO, E. O. et al. Propriedades de chapas produzidas com resíduos do fruto de coco e partículas de pinus. Floresta e Ambiente, v.11, n.2, p.1-6, 2004.

CARNEIRO, A. C. O. Efeito da hidrólise ácida e sulfitação de taninos de Eucalyptus grandis w. hill ex maiden e Anadenanthera peregrina speg., nas propriedades dos adesivos. 2006. $182 \mathrm{f}$. Tese (Doutorado em Ciência Florestal) Universidade Federal de Viçosa, MG, 2006.

\section{GERMAN STANDARDS CIMMITTEE:}

Deutschen Normanausschuss. Specifications for particleboards. Holtz: 1971. (DIN 68761 (1)-1961 (3)).

IMAMURA, Y. et al. Dimensional stability and biological resistance of particleboard from acetylated albizzia wood particles. Bulletin of Wood Research Institute, n.76, p.49-58, 1989.
MALONEY, T. M. Modern particleboard e dry-process fiberboard manufacturing. San Francisco, Miller Freeman, 1993. 681p.

MEDEVED, S.; RESNIK, J. Influence o0f the acidity and size of beech particles on the hardening of the urea-formaldehyde adhesive. Acta Chimica Slovenica., n.51, p.353-360, 2004.

SILVA, G. A. et al. Umidade de equilíbrio de painéis de madeira. Revista Árvore, v.29, n.4, p.639-646, 2005.

SILVA, J. C. A madeira como matériaprima para a indústria moveleira. Viçosa, MG: Universidade Federal de Viçosa, 2006. 56p. Não Publicado

ZENID, G. J. et al. Mercado estimula produtos de madeira com valor agregado. Revista da Madeira, n.84, p.34-46, 2004.

SOUZA, D. A. Comportamento térmico das fibras vegetais (Cocos nucifera L.). 1999. 80f. Dissertação (Mestrado em Química) Universidade Federal de Minas Gerais, Belo Horizonte, 1999.

SOUZA, D. B.; CARVALHO, G. S.; RAMOS, E. J. A. Paricá (Schizolobium amazonicum Huber ex Ducke. Belém: Rede de Sementes da Amazônia, 2005. 2p. (Informativo Técnico)

PASSOS, P. R. A. Destinação sustentável de cascas de coco (Cocos nucifera) verde: Obtenção de telhas e chapas de partículas. 2005. 185f. Tese (Doutorado em Ciências em Planejamento Energético) Universidade Federal do Rio de Janeiro, Rio de Janeiro, 2005.

VITAL, B. R. Effects of species and panel densities on properties of hardwood particleboard. 1973. 111p. (M.S.) - University of Wisconsin, Madison, 1973.

VITAL,B. R.; HASELEIN, C. R.Qualidade de chapas de aglomerado produzidas com embaúba (Cecropia sp) e bambu (Bambusa vulgaris). Revista Árvore, v.12, n.2, p.134-145, 1988. 